\title{
ASO Author Reflections: Neoadjuvant Endocrine Therapy: A Pill in Time Saves Nine
}

\author{
Austin D. Williams, MD, MSEd ${ }^{1}$, and Richard J. Bleicher, MD $^{2}$ \\ ${ }^{1}$ Department of Surgery, Lankenau Medical Center, Wynnewood, PA; ${ }^{2}$ Department of Surgical Oncology, Fox Chase \\ Cancer Center, Philadelphia, PA
}

\section{PAST}

The incidence of ductal carcinoma in situ (DCIS) has increased since screening mammography has become more widespread, leading to many more surgical excisions for non-invasive disease. ${ }^{1}$ However, the identification and treatment of pre-invasive disease has not decreased the rate of invasive or advanced breast cancers diagnosed over the same time period. This, and the degree of complexity related to the transformation of DCIS to invasive ductal carcinoma (IDC), have led to the development of ongoing trials investigating whether endocrine therapy and surveillance is a safe alternative to surgical excision in hormone receptor-positive (HR+) DCIS. ${ }^{2}$ Additionally, the postponement of many elective surgeries during the initial wave of the coronavirus disease 2019 (COVID-19) pandemic provided an impetus to implement an underused strategy of neoadjuvant endocrine therapy (NET) in HR+ DCIS and IDC as a bridge to surgery. ${ }^{3}$ Our group previously demonstrated a higher upgrade rate from DCIS to IDC in longer surgical delays, ${ }^{4}$ therefore we were interested in whether NET modulates this difference.

\section{PRESENT}

Given that the data from the trials of nonoperative management and post-pandemic outcomes are not yet available, the impact of NET and its interaction with

(C) Society of Surgical Oncology 2021

First Received: 15 November 2021

Accepted: 15 November 2021;

Published Online: 6 January 2022

R. J. Bleicher, MD

e-mail: richard.bleicher@fccc.edu surgical delay in patients with HR+ DCIS remains unknown. We sought to investigate whether there is an interaction between surgical delay and the use of NET in $\mathrm{HR}+$ DCIS in a prepandemic cohort, in order to determine whether NET limits progression to IDC. ${ }^{5}$ As expected, only a small proportion $(0.3 \%)$ of patients in this cohort received NET, and its use and duration were not associated with upgrade to IDC when examined on univariate and multivariable analyses. However, we did find evidence of a potential protective effect when stratifying by surgical delay, where the rate of upgrade increased for patients who did not receive NET, but did not increase in patients who received NET.

\section{FUTURE}

NET was uncommonly used in patients with DCIS prior to the pandemic, and we still cannot distinguish between DCIS that will remain indolent and that which becomes invasive. But if NET limits progression, as our study suggests, it may help combat the latter. With larger numbers of patients in contemporary prospective cohorts, it will be important to investigate the onset of action of NET, the magnitude and durability of the protective effect, and whether progression to invasion can be partly or even completely controlled. Such a capability would truly turn DCIS into a risk factor, and add new meaning to the expression 'an ounce of prevention is worth a pound of cure'.

\section{REFERENCES}

1. Bleyer A, Welch HG. Effect of three decades of screening mammography on breast-cancer incidence. $N$ Engl J Med. 2012;367(21):1998-2005. https://doi.org/10.1056/NEJMoa 1206809 . 
2. Kanbayashi C, Thompson AM, Hwang E-SS, et al. The international collaboration of active surveillance trials for low-risk DCIS (LORIS, LORD, COMET, LORETTA). J Clin Oncol. 2019;37(15 Suppl):TPS603. https://doi.org/10.1200/jco.2019.37.15_suppl. tps603.

3. Dietz JR, Moran MS, Isakoff SJ, et al. Recommendations for prioritization, treatment, and triage of breast cancer patients during the COVID-19 pandemic. The COVID-19 pandemic breast cancer consortium. Breast Cancer Res Treat. 2020;181(3):487-97. http s://doi.org/10.1007/s10549-020-05644-z.

4. Ward WH, DeMora L, Handorf E, et al. Preoperative delays in the treatment of DCIS and the Associated Incidence of Invasive Breast
Cancer. Ann Surg Oncol. 2020;27(2):386-96. https://doi.org/10. 1245/s10434-019-07844-4.

5. Williams AD, Chang C, Sigurdson ER, et al. Neoadjuvant endocrine therapy and delays in surgery for ductal carcinoma in situ: implications for the coronavirus pandemic. Ann Surg Oncol. 2021. https://doi.org/10.1245/S10434-021-10883-5.

Publisher's Note Springer Nature remains neutral with regard to jurisdictional claims in published maps and institutional affiliations. 